OPEN ACCESS

Edited by:

lliana Bersani,

Bambino Gesù Children Hospital (IRCCS), Italy

Reviewed by:

Anne Lee Solevåg,

Oslo University Hospital, Norway

Paola Elisa Cogo,

University of Udine, Italy

*Correspondence:

Fiammetta Piersigill

fiammetta.piersigilli@uclouvain.be

Specialty section: This article was submitted to Neonatology,

a section of the journa

Frontiers in Pediatrics

Received: 01 March 2021

Accepted: 24 May 2021

Published: 25 June 2021

Citation:

Debuf MJ, Carkeek K and Piersigilli F (2021) A Metabolomic Approach in

Search of Neurobiomarkers of Perinatal Asphyxia: A Review of the

Current Literature.

Front. Pediatr. 9:674585

doi: 10.3389/fped.2021.674585

\section{A Metabolomic Approach in Search of Neurobiomarkers of Perinatal Asphyxia: A Review of the Current Literature}

\author{
Marie Julie Debuf, Katherine Carkeek and Fiammetta Piersigilli* \\ Division of Neonatology, Cliniques Universitaires Saint Luc, Université Catholique de Louvain, Bruxelles, Belgium
}

Perinatal asphyxia and the possible sequelae of hypoxic-ischemic encephalopathy $(\mathrm{HIE})$, are associated with high morbidity and mortality rates. The use of therapeutic hypothermia $(\mathrm{TH})$ commencing within the first $6 \mathrm{~h}$ of life-currently the only treatment validated for the management of HIE-has been proven to reduce the mortality rate and disability seen at follow up at 18 months. Although there have been attempts to identify neurobiomarkers assessing the severity levels in HIE; none have been validated in clinical use to date, and the lack thereof limits the optimal treatment for these vulnerable infants. Metabolomics is a promising field of the "omics technologies" that may: identify neurobiomarkers, help improve diagnosis, identify patients prone to developing HIE, and potentially improve targeted neuroprotection interventions. This review focuses on the current evidence of metabolomics, a novel tool which may prove to be a useful in the diagnosis, management and treatment options for this multifactorial complex disease. Some of the most promising metabolites analyzed are the group of acylcarnitines: Hydroxybutyrylcarnitine (Malonylcarnitine) [C3-DC (C4$\mathrm{OH}$ ]), Tetradecanoylcarnitine [C14], L-Palmitoylcarnitine [C16], Hexadecenoylcarnitine [C16:1], Stearoylcarnitine [C18], and Oleoylcarnitine [C18:1]. A metabolomic "fingerprint" or "index," made up of 4 metabolites (succinate $\times$ glycerol// $\beta$-hydroxybutyrate $\times$ O-phosphocholine)), seems promising in identifying neonates at risk of developing severe HIE.

Keywords: neonate, perinatal asphyxia, hypoxic-ischemic encephalopathy, neurobiomarkers, metabolomics

\section{INTRODUCTION}

Perinatal asphyxia is defined as the deprivation of oxygen occurring around the time of birth, and despite improved antenatal and peripartum care, it remains one of the most important causes of infant morbidity and mortality $(1,2)$. Its worldwide incidence is estimated at 20/1,000 births (3) but varies strongly from region to region, with resource-limited countries having a more significant incidence. Even in countries with the highest levels of healthcare, 1-5/1,000 full-term infants develop hypoxic-ischemic encephalopathy (HIE), an encephalopathy indisputably attributed to hypoxic brain injury (4).

HIE is associated with a high death rate and long-term morbidity (5). It has been proven and widely accepted that the use of therapeutic hypothermia (TH) performed within the first $6 \mathrm{~h}$ of life for a duration of $72 \mathrm{~h}$, is associated with a significant reduction in death and disability at 18 months 
(6-11). This is currently the only treatment modality validated in moderate and severe HIE cases. TH is not currently standard treatment in mild encephalopathy; and a percentage of these neonates may, albeit with mild symptoms at birth, develop disabilities or cognitive abnormalities. TH may indeed be beneficial in these mild cases.

The discovery and validation of neurobiomarkers could be useful and add value as a screening tool, to stratify the different severity grades of HIE, and objectively assist in the criteria for TH. Furthermore, having diagnostic and prognostic biomarkers of severity, may lead to a more proactive approach in the use of adjunctive therapies other than hypothermia, such as melatonin or erythropoietin $(12,13)$. Ideal screening biomarkers should be easy to collect, have a fast laboratory turnaround time, be reliable and inexpensive. Current research on micro-RNAs, certain proteins and single metabolites has not identified an ideal biomarker fulfilling these criteria.

Metabolomics; defined as the study of low molecular weight metabolites present in the body, seems to be a promising novel approach in the search of neurobiomarkers. Metabolites analyzed may be peptides, lipids, organic acids, vitamins, minerals, drugs, amino acids, nucleic acids, carbohydrates, fatty acids, hormones, drugs, and any other chemicals with a molecular weight $<2,000$ Da. Metabolomic identification can be achieved using two techniques: a targeted and a non-targeted approach. The first approach consists of investigating pre-known and expected metabolites. The non-targeted approach conversely investigates all metabolites detectable in a sample, aiming to capture as much information as possible, providing a functional "fingerprint" of the pathological state being investigated. This metabolic fingerprint may then be validated in different experimental groups as a screening biomarker (14).

The use of metabolomics in HIE is 2-fold: firstly, adding to the understanding of biological pathways involved in HIE, and secondly creating a panel of metabolites that may act as this "fingerprint" characterizing the levels of severity in various HIE cases.

Several metabolomic studies have been performed over the last years studying various biological fluids. No specific neurobiomarkers to date have been validated for clinical use. The aim of this review is to analyze the most promising studies in the field of neurobiomarkers arising from metabolomics, in neonatal $\mathrm{HIE}$, in order to improve diagnosis and prognostication in this group of infants.

\section{METHODS}

\section{Search Strategy and Selection Criteria}

The review was conducted through a database search of PubMed and the Cochrane Library from January 1976 to February 2021.

The first search included the terms "metabolomics" AND "neonatal asphyxia" OR "perinatal asphyxia" OR "HIE" OR "hypoxic ischemic encephalopathy" OR "hypoxic ischemic

Abbreviations: LC-MS/MS, liquid chromatograpy-tandem mass spectrometry; HIE, hypoxic ischemic encephalopathy; $1 \mathrm{H}$ NMR, proton nuclear magnetic resonance; $\mathrm{TH}$, therapeutic hypothermia.

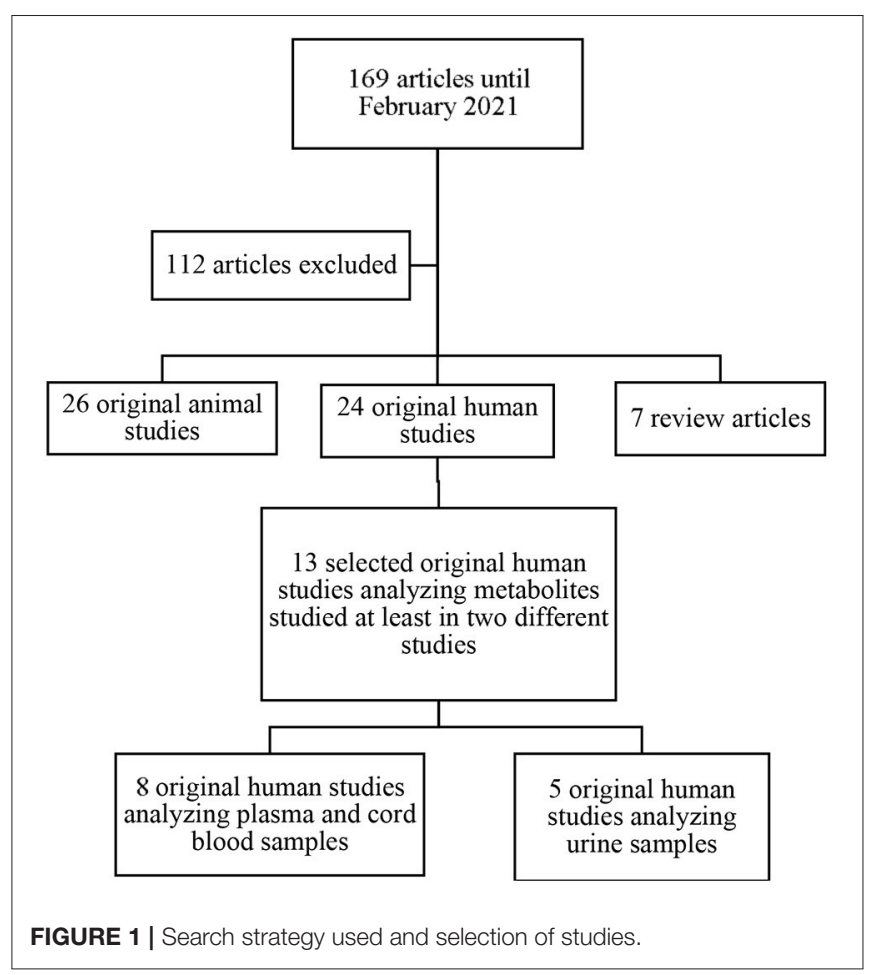

encephalopathy." The second search included the terms "neurobiomarkers" AND "neonatal asphyxia" OR "perinatal asphyxia" OR "hypoxic ischemic encephalopathy" OR "hypoxic ischemic encephalopathy." Only English-language, peerreviewed studies were included. Case reports and conference abstracts were excluded. We considered the studies eligible if they included term/late preterm infants ( $\geq 35$ weeks gestational age) diagnosed with perinatal asphyxia.

\section{Data Extraction}

The search identified 169 potentially relevant papers fitting the search criteria. After title and abstract screening, 57 fulltext studies were eligible for inclusion (Figure 1). Of the 57 studies included; 7 were review articles, 24 were original human studies, and 26 were original animal studies. We excluded the 7 review papers. After exclusion of articles not meeting inclusion criteria, we included 13 original human studies. The methods of metabolomic analysis are presented in Table $\mathbf{1 .}$

Animal studies were taken into consideration in order to support or qualify the findings from human studies.

It is clear from the literature and the reviews that an extensive number of biomarkers and metabolites have been studied. There remains no consensus however on the specific use of these neurobiomarkers in clinical practice.

It is important to note that the review articles already existing, included animal studies and these results may not be significant or translatable in humans (15-17). One review presented a range of biomarkers including RNA and cytokines and did not focus on metabolites specifically (18). The most recent review by Bardanzellu and Fanos (19) in 2020 reviewed the use of 
TABLE 1 | Studies assessing metabolites as biomarkers to predict neurological outcome in asphyxic neonates with/without HIE.

\begin{tabular}{|c|c|c|c|c|c|}
\hline \multirow{2}{*}{$\begin{array}{l}\text { Studied population } \\
\text { Neonates with HIE } \\
\text { (managed with } \\
\text { hypothermia) }\end{array}$} & \multicolumn{2}{|c|}{ Compared groups } & \multirow{2}{*}{$\begin{array}{l}\text { Timeline of the analysis } \\
48 \mathrm{~h} \text { after topiramate (TPM) } \\
\text { or placebo administration }\end{array}$} & \multirow{2}{*}{$\begin{array}{l}\text { Biological fluid } \\
\text { Plasma }\end{array}$} & \multirow{2}{*}{$\begin{array}{l}\text { Authors } \\
(22)\end{array}$} \\
\hline & HIE & Matched controls & & & \\
\hline & \multicolumn{2}{|c|}{ Metabolomic changes over time during HT } & $\begin{array}{l}\text { Birth, } 24,48 \text {, and } 72 \mathrm{~h} \text { after } \\
\text { the administration of TPM or } \\
\text { placebo }\end{array}$ & Cord blood + plasma & $(22)$ \\
\hline & Normal MRI day 4 to 8 & $\begin{array}{l}\text { Pathological MRI at day } 4 \\
\text { to } 8\end{array}$ & $\begin{array}{l}\text { Birth, } 24,48 \text {, and } 72 \mathrm{~h} \text { after } \\
\text { the administration of TPM or } \\
\text { placebo }\end{array}$ & Cord blood + plasma & $(46)$ \\
\hline \multirow{9}{*}{$\begin{array}{l}\text { Asphyxic Neonates } \\
\text { (No HIE diagnosis) }\end{array}$} & Asphyxia without HIE & Matched controls & Birth & Cord blood serum & $(29,31,54,55)$ \\
\hline & HIE without asphyxia & Matched controls & Birth & Cord blood serum & $(29,31,54,55)$ \\
\hline & Asphyxia with HIE & Matched controls & Birth & Cord blood dried spot & (56) \\
\hline & Asphyxia without HIE & HIE without asphyxia & Birth & Cord blood dried spot & (56) \\
\hline & Asphyxia without HIE & HIE without asphyxia & Birth & Cord blood serum & $(31,54)$ \\
\hline & HIE grade 3 & Matched controls & Birth & Cord blood serum & (29) \\
\hline & HIE grade 3 & Asphyxia without HIE & Birth & Cord blood serum & (29) \\
\hline & HIE severity grade & & Birth & Cord blood serum & $(29,54)$ \\
\hline & Norma/several outcome & & Birth & Cord blood serum & $(47)$ \\
\hline \multirow[t]{7}{*}{$\begin{array}{l}\text { Neonates with HIE } \\
\text { (managed with } \\
\text { hypothermia) }\end{array}$} & T1 (48h) & $\begin{array}{l}\text { Baseline = sample at } \\
\text { birth }=\text { T0 }\end{array}$ & $\begin{array}{l}5 \text { samples: birth (T0), after } \\
48 \mathrm{~h} \text { HT (T1), after } 72 \mathrm{~h} \mathrm{HT} \\
\text { (T2), after } 1 \mathrm{w} \text { (T3), after } 1 \mathrm{~m} \\
\text { (T4) }\end{array}$ & Urine & $(50)$ \\
\hline & T2 (72 h) & $\begin{array}{l}\text { Baseline = sample at } \\
\text { birth }=\text { TO }\end{array}$ & & & \\
\hline & T3 (1 week) & $\begin{array}{l}\text { Baseline = sample at } \\
\text { birth = T0 }\end{array}$ & & & \\
\hline & T4 (1 month) & $\begin{array}{l}\text { Baseline = sample at } \\
\text { birth }=\text { TO }\end{array}$ & & & \\
\hline & HIE at birth & Healthy controls at birth & $\begin{array}{l}4 \text { samples: birth, day } 2 \text {, day } \\
3 \text {, day } 30\end{array}$ & Urine & (49) \\
\hline & HIE at day 3 & HIE at birth & & & \\
\hline & HIE at day 30 & HIE at birth & & & \\
\hline \multirow[t]{4}{*}{$\begin{array}{l}\text { Asphyxic Neonates } \\
\text { (No HIE diagnosis) }\end{array}$} & $\begin{array}{l}\text { Asphyxia and HIE or } \\
\text { perinatal death }\end{array}$ & $\begin{array}{l}\text { Asphyxiated (without HIE) } \\
\text { and controls }\end{array}$ & $\begin{array}{l}2 \text { samples: within } 12 \mathrm{~h} \text { and } \\
\text { between } 12 \text { and } 48 \mathrm{~h}\end{array}$ & Urine & (69) \\
\hline & Asphyxia without HIE & $\begin{array}{l}\text { Asphyxiated and HIE or } \\
\text { perinatal death and } \\
\text { controls }\end{array}$ & & & \\
\hline & Asphyxia with HIE & Matched controls & 2 samples: day 1 , day 2 & Urine & $(48)$ \\
\hline & HIE with/without HT & Matched controls & $\begin{array}{l}3 \text { samples: day } 1 \text {, day } 3 \text {, day } \\
9\end{array}$ & Urine & (53) \\
\hline
\end{tabular}

metabolomics in pediatrics, however did not focus specifically on infants with asphyxia and encephalopathy due to hypoxicischemia. The two reviews "most similar" to our review, were published in 2010 and 2015, respectively $(3,20)$, and in an ever changing and dynamic research setting; we believe that there have been advances in techniques and interpretation worth reviewing.

In our review, we included human studies only with specific metabolites analyzed in at least two different studies. This method was chosen not only to narrow the spectrum of metabolites presented, but also to exclude possible nonsignificant metabolites found by chance. By doing so we feel this "fine-tuned" the proposed metabolites suggested for the screening process in asphyxiated infants. This proposed screening "tool" could be used/tested within the first hours of life in infants with asphyxia to ascertain which infants would develop encephalopathy and which would develop long-term poor outcome.

To our knowledge, this is the first review to present to clinicians and researchers, the metabolites across at least two studies show the greatest promise in differentiating and predicting the severity level and prognosis of infants born with asphyxia and hypoxic ischemic encephalopathy. 


\section{RESULTS}

The characteristics of the studies included are reported in Table 1, with an outline of the various metabolites considered as potential neurobiomarkers in HIE.

Amongst the 13 articles reviewed, two analyzed biological data of patients included in the HYPOTOP trial cohorta randomized, controlled, multicenter, double-blinded clinical trial aiming to assess the efficacy of topiramate vs. placebo in patients with HIE undergoing TH (21). Sanchez-Illana et al. (22) performed a study on this cohort analyzing metabolites involved in neurological pathways and they did not find any effect of the administration of topiramate on metabolite levels. It is for this reason we decided to include patients involved in the HYPOTOP trial in our review.

The most common metabolites considered as potential biomarkers, include energy related metabolites (lactate, pyruvate, glycerol, $\mathrm{N}$-acetylglucosamine, glucose, galactose, and lactose), amines, amino acids and amino acid metabolism intermediates (acylcarnitines, ketone bodies, metabolites of the Krebs cycle, steroid hormone biosynthesis metabolites), and other metabolites measured in urine (aconitate, formate, and kynurenic acid). The various metabolites/potential neurobiomarkers in HIE are described in detail below.

\section{Energy Related Key Metabolites Lactate}

Lactic acid is the final breakdown product of pyruvate during anaerobic metabolism. Lactate levels rise during hypoxia due to the shift from the aerobic metabolism to anaerobic metabolism. Lactate has been used for decades as a marker of poor tissue perfusion in newborns suffering from asphyxia and is highlighted in many studies performed both in humans (22-24) and animals (25-28).

\section{Blood Levels}

Blood lactate levels are significantly higher amongst newborns suffering from asphyxia (with or without signs of HIE) compared with controls $(22,29-31)$. It has been suggested that a lactate concentration of $>8 \mathrm{mmol} / \mathrm{L}$ is a good indicator of intrapartum asphyxia when associated with a base deficit of $>-12 \mathrm{mmol} / \mathrm{L}$ (32). Lactate levels alone however do not distinguish between asphyxiated neonates who develop HIE from those who recover $(29,31)$. Solberg et al. described in a population of newborn piglets, that the plasma lactate levels do not always correlate with hypoxia duration and significance (33).

Da Silva et al. established that a blood lactate level $>9 \mathrm{mmol} / \mathrm{L}$ at $30 \mathrm{~min}$ of life was associated with a moderate to severe clinical neurologic evolution (according to Finer's classification) with a sensitivity of $84 \%$ and a specificity of $67 \%$ (34). A level $<5$ $\mathrm{mmol} / \mathrm{L}$ was however associated with no risk of developing clinical signs of encephalopathy (35). In contrast, a study performed by Murray et al. showed that the initial rise of blood lactate observed in neonates with asphyxia could not be used to predict the gravity of the subsequent encephalopathy (36).

The persistence of a lactic acidosis has however been associated with severe encephalopathic patterns on EEG monitoring. After an initial increase, the lactate level should decrease gradually and normalize $(22,37)$. Shah et al. demonstrated that lactate levels normalize slower in asphyxiated newborns with moderate to severe encephalopathy in comparison to those presenting with mild encephalopathy (38). Even in asphyxiated lambs prolonged lactic acidosis was associated with important brain injury $(39,40)$. The clearance delay could be related to hepatic and renal dysfunction (41-44), or additional production of lactate due to convulsive activity (36). In terms of prognosis, Frey et al. showed that asphyxiated neonates who died during the first week of life had significantly higher lactate levels than those presenting with more mild neurological deficits (45). Pineiro-Ramos et al. however, could not demonstrate a relation between the cord blood lactate levels of neonates presenting with HIE and the severity of pathological cerebral magnetic resonance imaging (MRI) performed between day 4 and 8 of life (46). Ahearne et al. also showed that cord blood lactate levels in asphyxiated neonates did not predict outcome at 3 years of age (47).

\section{Urinary Levels}

Some studies have highlighted a higher urinary level of lactate in asphyxiated neonates (with and without HIE) in comparison to controls $(48,49)$. Urinary lactate levels generally decrease progressively with time (up to 1 month after birth) in neonates suffering from HIE $(49,50)$.

\section{Cerebrospinal Fluid Levels}

Magnetic resonance spectroscopy (MRS) studies suggest that high blood lactate levels after neonatal asphyxia, arise from altered brain metabolism. In fact, in neonates with HIE who died or developed severe neurological disability, high levels of lactate were shown both on cerebral proton magnetic resonance spectroscopy (51) and on the cerebrospinal fluid measurements (23). Van Cappellen et al. detected the presence of significantly higher levels of lactate in the cerebrospinal fluid of severely asphyxiated fetal lambs in comparison with a control group (52).

In summary, the increase and especially the persistence of high blood lactate levels, seem to reflect the degree of fetal hypoxia, but do not give clear predictive information on the risk of developing an adverse neurological outcome (18). As stated by Bennet et al. (20), it remains difficult to differentiate patients on the moderate to severe encephalopathy spectrum.

\section{Pyruvate \\ Blood Levels}

Reinke et al. observed that pyruvate cord blood levels were higher in asphyxiated neonates, with or without HIE compared to controls (29). Pyruvate levels did not however predict the severity of the grade of asphyxia, as the authors could not find a significant difference between neonates presenting with mild, moderate and severe HIE (Sarnat 3) compared to the control group. Even in the HYPOTOP trial, the analysis of pyruvate cord blood levels among neonates presenting with HIE and treated with TH, did not identify and differentiate those with pathological brain MRI findings (46). There were higher pyruvate levels $48 \mathrm{~h}$ after the administration of topiramate in neonates presenting with HIE 
and treated with $\mathrm{TH}$, in comparison to the control group. The level of pyruvate seems to decrease gradually during TH (22) and in an animal study (27) pyruvate blood levels were indeed higher $2 \mathrm{~h}$ post the asphyxic episode, decreasing progressively $4 \mathrm{~h}$ after the event.

In the HYPOTOP study, the pyruvate levels collected at 24 and $48 \mathrm{~h}$ in patients with HIE treated with $\mathrm{TH}$, did not predict the extent of pathology on cerebral MRI performed between day 4 and 8 of life. In samples collected $72 \mathrm{~h}$ after the administration of topiramate, the pyruvate level was however significantly higher in the group with a pathologic MRI (46).

\section{Urinary Levels}

Locci et al. found the urine pyruvate levels at birth lower in neonates presenting with HIE, in comparison to a control group (49). On the contrary, on the third and 30th day of life, the neonates with HIE treated with TH had higher urinary pyruvate level compared with levels measured at birth. Sarafidis et al. also observed higher urinary pyruvate levels in newborns with HIE (treated and non-treated with $\mathrm{TH}$ ), compared to controls at 3 days of life (53).

\section{Glycerol \\ Cord Blood Levels}

Two studies observed glycerol cord blood levels higher in patients with asphyxia (with or without HIE) than controls $(29,54)$. Reinke et al. (29) found that glycerol levels were significantly higher in neonates with asphyxia compared to controls, but only in the severe HIE (grade 3) subset. Similar findings were observed in an animal study performed on macaques (28). On the contrary, Denihan et al. (54) found no difference in the glycerol levels despite the severity of HIE.

\section{$\mathrm{N}$-Acetylglucosamine}

Studies measuring urinary $\mathrm{N}$-acetylglucosamine are discordant. Noto et al. studied neonates with HIE (treated with $\mathrm{TH}$ ) and found lower levels of urinary $\mathrm{N}$-acetylglucosamine measured at 48 and $72 \mathrm{~h}$, in comparison to levels measured at birth (50). The opposite was demonstrated by Locci et al., where a significant increase was found on day 3 and 30 of life in comparison to birth levels (49). In neonates with HIE, the levels measured at birth were lower than in the healthy control group.

\section{Glucose}

The studies performed by Sachse et al. and Longini et al. highlight significant glycosuria in asphyxiated patients (with or without HIE) in comparison with control groups (levels were carried out daily for the first 2 days of life) $(27,48)$. Locci et al. did not confirm this finding at birth, however urinary glucose was higher on the third day of life in patients with HIE (49). It is speculated that the asphyxic event leads to glycosuria, and although may not be evident in the first day of life, may appear in the following days.

\section{Galactose and Lactose}

These two metabolites can be analyzed in the urine. Noto et al. have shown in neonates with HIE treated with $\mathrm{TH}$, that levels were lower when measured after $72 \mathrm{~h}, 1$ week, and 1 month of life for galactose; and after $48 \mathrm{~h}, 1$ week, and 1 month of life for the lactose; in comparison to levels measured at birth (50). Locci et al. found the opposite finding (49) and no differences at birth between neonates with HIE and the control group.

In summary, concerning these energy related metabolites; pyruvate, glycerol, $\mathrm{N}$-acetylglucosamine, glucose, galactose, and lactose, several studies have demonstrated different levels between asphyxiated neonates and healthy controls, and none of the levels (collected in blood or urine and carried out at birth or later) showed significance in relation to the severity of asphyxia.

Regarding prognosis in HIE, only blood pyruvate levels (72 $\mathrm{h}$ after administration of the topiramate in the HYPOTOP study) allowed some prediction of cerebral MRI outcome, performed between day 4 and 8 of life.

\section{Amines, Amino Acids, and Amino Acid Metabolism Intermediates Blood Levels}

Amino acid levels analyzed from cord blood have been observed to be significantly higher in patients suffering from asphyxia in comparison to control groups. Among these amino acids according to various studies, alanine, creatinine, isoleucine, leucine, methionine, phenylalaline, taurine, and valine are the most significant (cf. Table 2) (29, 31, 54-56). The same finding has been observed in several animal studies $(25,27,28$, 33). Of note, only methionine levels showed the same trend systematically across the different studies and only alanine was found to be significantly higher in patients with severe HIE (grade 3 ) in comparison to asphyxiated patients without HIE and the control groups (29). Some amino acid levels were also significantly different amongst asphyxiated neonates who went on to develop HIE vs. those who did not $(29,56)$. The levels of alanine, phenylalanine, valine and leucine were significantly higher in the HIE group studied by El-Farghali et al. (56).

No difference was observed with the levels of creatinine, isoleucine and the methionine.

\section{Urinary Levels}

In the study of Longini et al. (48), urinary aspartate and threonine levels measured within the first 2 days of life were significantly higher in asphyxiated neonates (with and without HIE) in comparison with a control group. In contrast, Sarafidis et al. (53) found that patients with HIE (treated and not treated with $\mathrm{TH}$ ), had lower urinary levels of these two urinary amino acids on the first day of life compared to control group. On the contrary, no difference was highlighted between those two groups when the levels were measured after 3 and 9 days of life (53).

Longini et al. found the urinary levels of betaine, creatinine and dimethylglycine significantly lower in asphyxiated children (with or without HIE) in comparison to a control group during the two first days of life (48). However, differences have been highlighted in other publications between these 3 metabolites. Noto et al. showed an increase of urinary creatinine in neonates presenting with HIE (treated with $\mathrm{TH}$ ) at the end of treatment and at 1 week of life (50) and Locci et al. found increases only in betaine at birth in patients presenting with HIE in comparison to the control group (49). In this study, the evolution of betaine in neonates with HIE treated with $\mathrm{TH}$ is characterized by a decrease 
TABLE 2 | Variation of cord blood metabolites.

\begin{tabular}{|c|c|c|c|}
\hline Biomarker & References & $\begin{array}{l}\text { Levels } \\
\text { associated } \\
\text { with Asphyxia }\end{array}$ & $\begin{array}{l}\text { Levels } \\
\text { associated } \\
\text { with HIE }\end{array}$ \\
\hline Lactic acid & $(22,31)$ & & \\
\hline Creatine & $(29,54)$ & & \\
\hline Creatinine & $(29,54)$ & & \\
\hline Carnitine & $(29,31,54)$ & & \\
\hline Alanine & $(29,55,56)$ & & \\
\hline Isoleucine & $(29,55)$ & & \\
\hline Isoleucine & $(29,55)$ & & \\
\hline Leucine & $(29,54-56)$ & & \\
\hline Methionine & $(29,31,55,56)$ & & \\
\hline Phenylalanine & $(29,31,54-56)$ & & \\
\hline Valine & $(29,55,56)$ & & \\
\hline L-acetylcarnitine [C2] & $(54-56)$ & & \\
\hline Hydroxybutyrylcarnitine & $(55,56)$ & & \\
\hline Butyrylcarnitine [C4] & $(55,56)$ & & \\
\hline $\begin{array}{l}\text { Tetradecanoylcarnitine } \\
\text { [C14] }\end{array}$ & $(55,56)$ & & \\
\hline L-Palmitoylcarnitine [C16] & $(55,56)$ & & \\
\hline $\begin{array}{l}\text { Hexadecenoylcarnitine } \\
\text { [C16:1] }\end{array}$ & $(55,56)$ & & \\
\hline Stearoylcarnitine [C18] & $(55,56)$ & & \\
\hline Oleoylcarnitine [C18:1] & $(55,56)$ & & \\
\hline Pyruvate & $(22,29,46)$ & & \\
\hline Hydroxybutyrate & $(22,29)$ & & \\
\hline
\end{tabular}

at day 3 of life followed by an increase at day 30 of life. Creatinine levels present an opposite evolution with an increase at day 3 of life and a decrease at day 30 of life (in comparison to the level measured at birth). The level of dimethylglycine however, remains elevated at day 3 and day 30 of life (in comparison to the level measured at birth).

Longini et al. (48) also analyzed dimethylamine in the urine during the first 2 days of life; and found the level to be significantly lower in asphyxiated neonates (with or without HIE) in comparison to a control group. This tendency was confirmed by Locci et al. (49), demonstrating lower dimethylamine levels at birth in patients presenting a HIE in comparison to the control group. Within the same group of HIE infants, there was a significant increase of dimethylamine levels at the end of $\mathrm{TH}$ and at 30 days of life, in comparison to the level measured at birth.

Neonates with asphyxia and HIE have higher levels of urinary taurine at birth compared to controls. Locci et al. highlight a constant decline in levels during the $72 \mathrm{~h}$ of $\mathrm{TH}$ (49). Noto et al. observed the same tendency (50) with a decrease measured at 1 week and 1 month after birth.
In summary, although amines, amino acids and amino acid metabolism intermediate levels (carried out at birth and/or thereafter and measured in the blood and/or in urine), may show differences in levels between asphyxiated neonates and healthy controls across several studies, they do not predict severity or outcome in HIE.

\section{Acylcarnitines}

The acylcarnitines reviewed and mentioned are represented by their chemical nomenclature which is shown between brackets (e.g., Oleoylcarnitine is [C18:1]).

\section{Blood Levels}

Studies performed in animals have highlighted higher levels of acylcarnitines after hypoxia $(33,57)$. Similarly, the cord blood levels of several acylcarnitines were significantly higher in asphyxiated neonates in comparison to control groups (cf. Table 2).

In particular, L-acetylcarnitine [C2] and Butyrylcarnitine [C4] blood levels have been shown to be higher in asphyxiated neonates (with or without HIE) in comparison to control groups $(31,55,56)$. These levels did not however identify those at risk of developing HIE $(54,56)$ or predict the grade of severity of HIE in which the infant would be (54).

The level of Hydroxybutyrylcarnitine (Malonylcarnitine) [C3-DC (C4-OH)], Tetradecanoylcarnitine [C14], LPalmitoylcarnitine [C16], Hexadecenoylcarnitine [C16:1], Stearoylcarnitine [C18], and Oleoylcarnitine [C18:1] were not only significantly higher in asphyxiated patients in comparison with control groups $(31,55,56)$, but were also significantly higher in the group of asphyxiated patients who developed HIE in comparison to those who did not develop HIE (56).

\section{Ketone Bodies Blood Levels}

Levels of acetone and $\beta$-hydroxybutyrate analyzed on cord blood $(29,54)$ have been found to be higher in asphyxiated patients without HIE in comparison to the control group. Oddly however levels were not significantly different when comparing patients with HIE to the control group. Only in the study of Reinke et al. (29) were the levels of acetone and $\beta$-hydroxybutyrate measured on cord blood higher in neonates with severe HIE (grade 3) compared to asphyxiated neonates without HIE.

Furthermore, the HYPOTOP study revealed a significant decrease in the level of $\beta$-hydroxybutyrate $48 \mathrm{~h}$ after the injection of topiramate in the group of patients presenting with HIE and treated with $\mathrm{TH}$ in comparison to the control group (22). A progressive decrease was observed during $\mathrm{TH}$.

\section{Urinary Levels}

Urinary acetate levels, in the context of neonatal hypoxia, have been performed in the following two studies; Longini et al. and Locci et al. Longini et al. highlighted lower levels of ketone bodies in asphyxiated neonates (with or without HIE) in an analysis performed during the two first days of life, in comparison to a healthy control group (48). The same was found by Locci et al. in a cohort of neonates at birth presenting with HIE (49). 
In summary, even though the ketone body levels have differed in several studies between asphyxiated neonates and healthy controls, none of the considered levels (carried out at birth and measured on cord blood or later measured in urine) enabled prediction of the severity of HIE.

\section{Metabolites of the Krebs Cycle Blood Levels}

In the studies of Reinke et al. (29) and O'Boyle et al. (31), the succinate levels measured on cord blood were higher in asphyxiated neonates (with or without HIE) than in the control group. This finding has also been observed in several studies performed in animals $(27,28,33,58)$. Furthermore, a significant difference was found between asphyxiated neonates presenting with HIE (independent of grade) and those without HIE.

Denihan et al. however did not reach the same conclusion. Using untargeted metabolomics, they analyzed a metabolite with features similar to succinate (but also Methylmalonate or Threonolactone). This analysis revealed an increase of the metabolite among the group of asphyxiated neonates who did not develop HIE, but not in the group presenting with HIE (when compared to the control group) (54). Furthermore, even though these levels were statistically significant in the various severity groups of HIE, this difference did not remain significant following correction for confounding factors.

In the study of Sanchez-Illana et al. (HYPOTOP study) (22), there was no difference in the succinate levels measured $48 \mathrm{~h}$ after administration of topiramate in neonates presenting with HIE (treated with $\mathrm{TH}$ ), in comparison to the control group. The succinate levels in the HIE group remained stable during throughout $\mathrm{TH}$.

\section{Urine Levels}

Citrate and succinate levels have been studied in urine too. These two metabolites were found in lower levels in asphyxiated patients (with or without HIE) in comparison to the control group $(48,49)$.

In summary, even though differences in the levels of metabolites of the Krebs cycle were found in several studies, none of the observed levels predicted the severity of HIE.

\section{Steroid Hormone Biosynthesis Metabolites}

Among all the steroid hormone biosynthesis metabolites studied by Pineiro-Ramos et al. (46), only dehydroepiandrosterone had been analyzed in a second study by Denihan et al. (54). It is important however to note that the latter study was performed using untargeted metabolomics and the authors analyzed a metabolite with features matching dehydroepiandrosterone sulfate but also epitestosterone sulfate or testosterone sulfate.

Pineiro-Ramos et al. highlighted an increase in the levels of dehydroepiandrosterone, in neonates with HIE (treated with $\mathrm{TH}$ ), collected on cord blood and at 24,48 , and $72 \mathrm{~h}$ after the administration of topiramate (HYPOTOP study). These neonates had pathological MRIs between day 4 and 8 of life. Denihan et al. showed a significant decrease of dehydroepiandrosterone levels in asphyxiated neonates without HIE compared to a control group of neonates without asphyxia.
In summary, the limited number of studies analyzing dehydroepiandrosterone as a neurobiomarker, and the findings arising from these studies, do not validate its efficiency in differentiating neonates with HIE from control neonates. Furthermore, to our knowledge, no studies in animals have investigated this biomarker.

\section{Purines and Pyrimidine Derivatives}

Denihan et al. (54) and O'Boyle et al. (31) found a significant increase in uridine levels measured on cord blood of asphyxiated neonates with and without HIE, compared to a control group of neonates without asphyxia. Both studies however did not find any difference between asphyxiated patients with HIE and those without HIE, making the use of this metabolite less useful.

\section{Other Metabolites Measured in Urine Aconitate and Formate}

Longini et al. reported lower levels of aconitate and formate, two urinary metabolites, during the first 2 days of life, in asphyxiated neonates with or without HIE, in comparison to a control group. There was no difference however at birth between patients with HIE and the control group $(48,49)$. The levels were higher after 30 days of life (in comparison to the level measured at birth) in patients presenting with HIE and treated with TH (49). Even at 3 days of life, the aconitate levels were higher than birth levels in the HIE group (49). This was not seen in the formate levels.

\section{Kynurenic Acid}

Sarafidis et al. showed a lower level of kynurenic acid on the first day of life, in neonates presenting with HIE (with or without treatment with $\mathrm{TH}$ ), in comparison to a control group (53). This difference disappeared after the third day of life. Noto et al. highlighted a decrease in kynurenic acid levels at 48 and $72 \mathrm{~h}$, and 1 month of life in a population of neonates suffering from HIE and treated with $\mathrm{TH}$, in comparison with their levels measured at birth (50).

The limited number of studies analyzing this metabolite leaves unanswered questions regarding its utility.

In summary, neither aconitate and formate, nor kynurenic acid levels seem to accurately predict the degree of severity of asphyxia in infants with HIE.

\section{DISCUSSION}

The pathophysiological mechanisms behind HIE are complex, varied and at times not well-understood. Many factors such as the etiology, the duration and the severity of hypoxia, and the regional variations of cerebral blood flow can influence the nature and the extent of the brain injury as well as the outcome of the affected neonate. Animal models have allowed us to understand that the initial damage caused by the switch to anaerobic metabolism (primary energy failure) produces an immediate cell death. The restoration of cerebral blood flow after the ischemic insult leads to an alteration of metabolism (the secondary energy failure) provoking an inflammatory cascade. This inflammatory phase is characterized by the production of reactive oxygen species (free-radicals), the formation of proteases 
and caspases and leads to excitotoxicity, apoptosis and neuronal cell death. This second phase occurs after a latent period of 6 to $48 \mathrm{~h}(59)$.

The pathophysiology of HIE is strongly dependent on the perturbation of the different mechanisms of compensation, which usually withstand the "physiological" hypoxia found during labor and delivery. The neonatal brain is highly dependent on oxygen and has a low concentration of antioxidants, but a high concentration of unsaturated fatty acids. Together, these characteristics make the neonatal brain vulnerable to permanent damage, if critical levels of energy deprivation are reached (60), leading to a wide spectrum of neurological deficits.

$\mathrm{TH}$ is the only evidenced based treatment available to date to reduce the incidence of neurological impairment due to asphyxia at birth. Hypothermia has proven to be effective especially in moderate to severe cases of asphyxia. Nevertheless, it is not always easy after the ischemic event, to determine and accurately predict in which severity group the neonates may be and especially in the infants with mild asphyxia, who may be at risk of developing neurological impairment. With this in mind, the aim of investigating and validating neurobiomarkers to identify neonates at risk is highly suitable.

Metabolomics is promising is the field of biomarker identification. It consists of the quantitative analysis of many low molecular mass metabolites, involving both substrates and products of particular metabolic pathways, which may identify key metabolite levels (raised or decreased) due to the intricate interaction between the pathophysiological state, gene expression, and environment.

A study performed in adults highlighted that hypoxia has a dose-dependent effect on the metabolic composition in urine (61). It is intuitive that in cases of severe hypoxia there will be a modification of the metabolic milieu. In cases of severe neonatal hypoxia, the use of neurobiomarkers may seem less attractive, as TH is clearly indicated in these severe cases. It is however in the cases with mild hypoxia, where the current treatment guidelines do not promote/advocate for $\mathrm{TH}$, that studies are now reporting in the region of $20 \%$ of cases having adverse neurological outcomes at a later stage (62). Brain imaging of these infants with mild encephalopathy may show detectable abnormalities on MRI $(63,64)$. It is therefore becoming more evident that the grade of neonatal encephalopathy during the first hours of life does not always adequately identify the children prone to develop cerebral injury.

Different biofluids can be used for the identification of neurobiomarkers. Blood measurements allow fast and often realtime information, but blood collection in neonates may be difficult to perform and remains relatively invasive given their low total blood volume. Cord blood is easy to collect and is available in large volumes allowing multiple analyses, however only analyzes the situation at the moment of delivery. The studies of cord blood biomarkers combined with subsequent blood plasma analysis prove to provide a more interesting and inclusive approach.

The collection of urine in theory is easy and non-invasive, but may depend on the renal function of the neonate and in particular, infants with perinatal asphyxia are often oliguric or anuric and it may therefore take several hours to collect sufficient quantities of urine. This could represent a problem, as the goal of the neurobiomarker identification is (within the first $6 \mathrm{~h}$ of life) to ascertain whether or not the infant will benefit from TH and to expedite the treatment.

The evidence of analyzing saliva as a biofluid in neonates is sparse in the literature, and although it has the advantage of being easy to collect and non-invasive, it may be limited in quantity at times.

The analysis of cerebrospinal fluid may be crucial for the diagnosis and management of certain neurological diseases. Lumbar punctures are however invasive and not always easy to perform in neonates. The quantity of fluid obtained may also be limited.

There are indeed numerous metabolites that have been studied, reviewed and reported as proposed neurobiomarkers. Skappak et al. highlighted that whilst they found 13 urinary metabolites associated with hypoxia in piglets (in comparison to the control group), if these were studied independently, the metabolites were not found to be significant. When the 13 metabolites were combined however, they had an accuracy of $90 \%$ (25). This reiterates that often the most effective way to interpret metabolomics, is through the identification of a particular "fingerprint," composed of various metabolites expressed together, in a clinical setting such as asphyxia.

Many studies have shown that hypoxic neonates exhibit increased metabolites derived from lipid peroxidation (65). These measurements however, have appeared to be ineffective in identifying those who will develop HIE. A common finding across studies was that neonates with severe HIE are unable to produce neuroprotective ketones $(22,29)$. The metabolites proving to be most promising in identifying neonates who will develop HIE, are the group of acylcarnitines: Hydroxybutyrylcarnitine (Malonylcarnitine) [C3-DC (C4$\mathrm{OH})]$, Tetradecanoylcarnitine [C14], L-Palmitoylcarnitine [C16], Hexadecenoylcarnitine [C16:1], Stearoylcarnitine [C18], and Oleoylcarnitine [C18:1]. In the studies of Walsh and El-Farghali the metabolites mentioned above were not only significantly higher in the cord blood of asphyxiated neonates compared to normal controls, but were significantly higher in the group presenting with HIE compared to the group of asphyxiated patients who did not present with $\operatorname{HIE}(55,56)$. From a biological point of view, this could be explained by the reduced level of $\beta$-oxidation of long chain acyl-CoA due to the occurrence of hypoxia, which seems to induce the accumulation of its precursor: acylcarnitines (16). This early screening measurement thus seems promising, as it may allow early identification of neonates who would benefit from $\mathrm{TH}$.

A promising metabolomic "index," created to identify the neonates at risk of developing HIE, was proposed by Reinke et al. In their study, several metabolites associated with HIE, were measured on cord blood, and stepwise multiple linear regression showed that 3-hydroxybutyrate, glycerol, O-phosphocholine, and succinate were most predictive of HIE severity. The "index" used was the following: (succinate $\mathrm{x}$ glycerol) / ( $\beta$-hydroxybutyrate $\times$ O-phosphocholine) (29). 
This index was subsequently validated in a study by Ahearne et al. (47). They analyzed the index in relation to the outcome at 3 years of age in a population of asphyxiated neonates (including those with and without HIE). The 3 year outcome used was the Bayley Scales of Infant and Toddler Development, ed. III (BSIDIII). The "severe" outcome group was defined as those infants with the outcomes of death, dyskinetic, or spastic quadriplegic cerebral palsy or $\leq 70$ in all BSID-III subscales. The study revealed that a metabolite index of $<0.13$ was associated with normal outcome (sensitivity $65 \%$ and specificity of $91 \%$ ), whereas an index of $>2.4$ was associated with a "severe outcome" (sensitivity of $80 \%$ and specificity of $100 \%$ ). This index method needs to be validated on urine as due to the relative ease of collection, an increased sampling opportunity is available.

The use of this metabolite index is the first attempt combining several metabolites in order to analyze the blood metabolome in asphyxiated newborns. The study of these complex biomarker relationships may assist for diagnostic and prognostic purposes. This approach seems promising and validation in larger groups of asphyxiated neonates is needed to provide further evidence of its reliability, and its implication in predicting outcome in this vulnerable group of neonates.

Ahearne et al. (47) suggested that the biological plausibility of this metabolomic index increases the probability of its predictive potential. Indeed, hypoxia-ischemia leads to changes in cellular energy pathway molecules, such as glycerol and succinate, through mitochondrial dysfunction and subsequent disruption of the tricarboxylic acid cycle (33). O-phosphocholine is an anabolic and catabolic metabolite of cell membrane metabolism, and together with glycerol may indicate cell membrane degradation (66). The modification of the levels of O-phosphocholine has been previously described in animal models of hypoxia-ischemia (67). The ability of the brain to upregulate ketone metabolism to provide an alternative energy source is an important means of brain adaptation in early life (68). This is the reason why the contribution of hydroxybutyrate to the model may indicate defective ketone body production, complete consumption of the ketone body supply, or inadequate prior adaptation to ketone body metabolism, causing the insurmountable secondary energy failure leading to long-term damage (47).

Recently, O'Boyle et al. also tried to combine several metabolites in order to produce a snapshot of the expected blood metabolome in asphyxiated newborns at birth (31). Unfortunately, umbilical cord blood metabolites alone were not sufficiently predictive of the development of HIE in asphyxiated neonates. For this reason, they added clinical data to these measurements. In summary, they showed that the clinical condition at birth (Apgar scores at 1 and $5 \mathrm{~min}$, details of intensive resuscitation required at birth, and the need for assisted ventilation at $10 \mathrm{~min}$ of age), combined with both lactic acid and alanine measurements could be used to give the optimum model for distinguishing between neonates with perinatal asphyxia alone and those who would go on to develop HIE. Alanine plays a key role in gluconeogenesis in mammals and is also the result of the transamination of pyruvate (deriving from lactate). It is for this reason the increase is significant as it provides evidence of the transitional state to anaerobic metabolism. It is therefore not surprising that a rise in circulating alanine is seen in asphyxiated neonates. The significance of the increase of lactate during hypoxia has been explained previously.

\section{Limitations and Strengths}

In order to critically appraise this review, one needs to acknowledge the limitations and the strengths of the review. One of the main limitations is that the effects of $\mathrm{TH}$ itself on the metabolic pattern were not explored and discussed here. The decrease of body temperature is responsible for the slowing of the metabolism and can alter enzymatic activity and therefore the production of different metabolites. This may influence and lead to complex effects on multiple regulatory processes.

The feeding and infusion regimens of the neonates in the studies reviewed was also not discussed, both of which could affect the metabolic pattern. It is well-described and practiced that neonates undergoing $\mathrm{TH}$ are kept nil per os or with minimal enteral feeding and this effect on the metabolite results may be negligible. This may not be the case for parenteral nutrition.

It is true that whilst most of the reviewed articles measured the metabolites on cord blood at birth, there were some measurements within $72 \mathrm{~h}$ (and later). No specific mention was made of the feeding regimes. Receiving parenteral nutrition may have influenced these metabolite levels although most parenteral nutrition solutions used in the neonatal intensive care units have standardized protein, fat and carbohydrate components and are standardized for each day of life.

In the design of future studies, it would be important to take this factor into account by reviewing the volume $/ \mathrm{kg}$ of parenteral nutrition received and or other fluids/feeds received, when analyzing the data.

We are specifically interested in the metabolite levels within the first $6 \mathrm{~h}$ of life, as it is within this crucial window that the clinicians need to decide which infants would benefit from therapeutic hypothermia. At this early stage, parenteral nutrition has not generally been commenced. If one measured the metabolites in the infants with asphyxia at a later stage (for example day 2, 3 onwards), this would need to carefully considered.

Another important point which may be viewed as a limitation, is the inclusion of studies that analyzed data from the HYPOTOP trial cohort. The reasons for including these papers have been described. Finally, given the extensive number of metabolites studied in the current literature, the decision to review and include only the articles with metabolites analyzed in at least two different studies was decided arbitrarily, which may lead to bias or important missed metabolites not reviewed.

The review also presents strengths as the current literature has a significant number of metabolites studied in the context of HIE. To our knowledge, this review is the first to present to clinicians and researchers in a concrete manner, the metabolites that, according to several studies, seem to have the greatest ability to differentiate the degree of severity of asphyxia in these patients. 


\section{CONCLUSION}

There is currently early preliminary knowledge of the neurobiomarkers that may assist physicians in improving the selection criteria for therapeutic hypothermia after perinatal asphyxia. It appears these early validation results, using a panel of neurobiomarkers acting together as a "fingerprint," may add information to help categorize infants into HIE severity groups, improve and guide management with $\mathrm{TH}$, as well as aid as a prognostic tool.

Metabolomics, a novel methodology in the development of neurobiomarkers, ideally measured within the first $6 \mathrm{~h}$ of life, may be able to identify neonates who will develop, without further intervention, encephalopathy. Despite a bedside method to measure these metabolites not being currently available, considering the increasing and keen interest of metabolomics it seems realistic and possible for future "bench to the bedside" analysis advances. Not only will this add vital additional information for neonates considered as having "mild asphyxia" (where in most centers worldwide TH is not offered), but will lead to more prompt treatment in all neonates at risk. The

\section{REFERENCES}

1. Lawn JE, Cousens SJ. Zupan, Lancet Neonatal Survival Steering Team. 4 million neonatal deaths: when? Where? Why? Lancet. (2005) 365:891900. doi: 10.1016/S0140-6736(05)71048-5

2. Liu L, Oza S, Hogan D, Chu Y, Perin J, Zhu J, et al. Global, regional, and national causes of under-5 mortality in 2000-15: an updated systematic analysis with implications for the sustainable development goals. Lancet. (2016) 388:3027-35. doi: 10.1016/S0140-6736(16)31593-8

3. Denihan NM, Boylan GB, Murray DM. Metabolomic profiling in perinatal asphyxia: a promising new field. Biomed Res Int. (2015) 2015:254076. doi: 10.1155/2015/254076

4. Snowden JM, Cheng YW, Kontgis CP, Caughey AB. The association between hospital obstetric volume and perinatal outcomes in California. Am J Obstet Gynecol. (2012) 207:478 e1-7. doi: 10.1016/j.ajog.2012.09.029

5. Edwards AD, Brocklehurst P, Gunn AJ, Halliday H, Juszczak E, Levene M, et al. Neurological outcomes at 18 months of age after moderate hypothermia for perinatal hypoxic ischaemic encephalopathy: synthesis and meta-analysis of trial data. BMJ. (2010) 340:c363. doi: 10.1136/bmj.c363

6. Gluckman PD, Wyatt JS, Azzopardi D, Ballard R, Edwards AD, Ferriero DM, et al. Selective head cooling with mild systemic hypothermia after neonatal encephalopathy: multicentre randomised trial. Lancet. (2005) 365:66370. doi: 10.1016/S0140-6736(05)70932-6

7. Shankaran S, Laptook AR, Ehrenkranz RA, Tyson JE, McDonald SA, Donovan EF, et al. Human development neonatal research, whole-body hypothermia for neonates with hypoxic-ischemic encephalopathy. $N$ Engl J Med. (2005) 353:1574-84. doi: 10.1186/s13063-017-2165-3

8. Azzopardi DV, Strohm B, Edwards AD, Dyet L, Halliday HL, Juszczak E, et al. Moderate hypothermia to treat perinatal asphyxial encephalopathy. $N$ Engl J Med. (2009) 361:1349-58. doi: 10.1056/NEJMoa0900854

9. Simbruner G, Mittal RA, Rohlmann F. Muche R, neo.nEURO.network Trial Participants. Systemic hypothermia after neonatal encephalopathy: outcomes of neo.nEURO.network RCT. Pediatrics. (2010) 126:e7718. doi: 10.1542/peds.2009-2441

10. Zhou WH, Cheng GQ, Shao XM, Liu XZ, Shan RB, Zhuang DY, et al. China study, selective head cooling with mild systemic hypothermia after neonatal hypoxic-ischemic encephalopathy: a multicenter randomized controlled trial in China. J Pediatr. (2010) 157:367-72:372 e1-3. doi: 10.1016/j.jpeds.2010.03.030 dynamic rate of the biomarker evolution also will add additional information for prognostic value.

As mentioned, in order to produce and provide a safe and effective screening tool, the collection of samples needs to be easy, the analysis reliable and the costs kept to a minimum. As shown in this review, no single metabolite seems to fulfill these criteria. In the future, combinations of metabolites used as a biomarker "fingerprint" need to be further studies and in larger groups of neonates to validate these approaches. The relatively low numbers of neonates with HIE highlights the importance of multi-centric studies, to increase the numbers of neonates studied, pool knowledge and decrease the risk of inclusion bias. These considerations should be taken into account in future study designs.

\section{AUTHOR CONTRIBUTIONS}

$\mathrm{MD}$ and $\mathrm{FP}$ were responsible for reviewing the articles. $\mathrm{MD}$, $\mathrm{KC}$, and FP were responsible in writing the manuscript and for important intellectual content. All authors contributed to the article and approved the submitted version.

11. Jacobs SE, Morley CJ, Inder TE, Stewart MJ, Smith KR, McNamara PJ, et al. Infant cooling evaluation, whole-body hypothermia for term and near-term newborns with hypoxic-ischemic encephalopathy: a randomized controlled trial. Arch Pediatr Adolesc Med. (2011) 165:692-700. doi: 10.1001/archpediatrics.2011.43

12. Jerez-Calero A, Salvatierra-Cuenca MT, Benitez-Feliponi A, Fernandez-Marin CE, Narbona-Lopez E, Uberos-Fernandez J, et al. Hypothermia plus melatonin in asphyctic newborns: a randomized-controlled pilot study. Pediatr Crit Care Med. (2020) 21:647-55. doi: 10.1097/PCC.0000000000002346

13. Oorschot DE, Sizemore RJ, Amer AR. Treatment of neonatal hypoxicischemic encephalopathy with erythropoietin alone, and erythropoietin combined with hypothermia: history, current status, future research. Int J Mol Sci. (2020) 21:1487. doi: 10.3390/ijms21041487

14. Griffiths WJ, Koal T, Wang Y, Kohl M, Enot DP, Deigner HP. Targeted metabolomics for biomarker discovery. Angew Chem. (2010) 49:542645. doi: 10.1002/anie.200905579

15. Locci E, Bazzano G, Demontis R, Chighine A, Fanos V, d'Aloja E. Exploring perinatal asphyxia by metabolomics. Metabolites. (2020) 10:141. doi: 10.3390/metabo10040141

16. Fattuoni C, Palmas F, Noto A, Fanos V, Barberini L. Perinatal asphyxia: a review from a metabolomics perspective. Molecules. (2015) 20:700016. doi: $10.3390 /$ molecules 20047000

17. Efstathiou N, Theodoridis G, Sarafidis K. Understanding neonatal hypoxicischemic encephalopathy with metabolomics. Hippokratia. (2017) 21:115-23.

18. Murray DM. Biomarkers in neonatal hypoxic-ischemic encephalopathyreview of the literature to date and future directions for research. Handb Clin Neurol. (2019) 162:281-93. doi: 10.1016/B978-0-444-64029-1.00013-8

19. Bardanzellu F, Fanos V. How could metabolomics change pediatric health? Ital J Pediatr. (2020) 46:37. doi: 10.1186/s13052-020-0807-7

20. Bennet L, Booth L, Gunn AJ. Potential biomarkers for hypoxicischemic encephalopathy. Semin Fetal Neonatal Med. (2010) 15:253-60. doi: 10.1016/j.siny.2010.05.007

21. Nunez-Ramiro A, Benavente-Fernandez I, Valverde E, Cordeiro M, Blanco D, Boix $\mathrm{H}$, et al. Topiramate plus cooling for hypoxic-ischemic encephalopathy: a randomized, controlled, multicenter, double-blinded trial. Neonatology. (2019) 116:76-84. doi: 10.1159/000499084

22. Sanchez-Illana A, Nunez-Ramiro A, Cernada M, Parra-Llorca A, Valverde E, Blanco D, et al. Evolution of energy related metabolites in plasma 
from newborns with hypoxic-ischemic encephalopathy during hypothermia treatment. Sci Rep. (2017) 7:17039. doi: 10.1038/s41598-017-17202-7

23. Fernandez F, Verdu A, Quero J, Ferreiros MC, Daimiel E, Roche MC, et al. Cerebrospinal fluid lactate levels in term infants with perinatal hypoxia. Pediatr Neurol. (1986) 2:39-42. doi: 10.1016/0887-8994(86)90038-X

24. Leth H, Toft PB, Peitersen B, Lou HC, Henriksen O. Use of brain lactate levels to predict outcome after perinatal asphyxia. Acta Paediatr. (1996) 85:859-64. doi: 10.1111/j.1651-2227.1996.tb14168.x

25. Skappak C, Regush S, Cheung PY, Adamko DJ. Identifying hypoxia in a newborn piglet model using urinary NMR metabolomic profiling. PLoS ONE. (2013) 8:e65035. doi: 10.1371/journal.pone.0065035

26. Sanchez-Illana A, Solberg R, Lliso I, Pankratov L, Quintas G, Saugstad OD, et al. Assessment of phospholipid synthesis related biomarkers for perinatal asphyxia: a piglet study. Sci Rep. (2017) 7:40315. doi: 10.1038/srep40315

27. Sachse D, Solevag AL, Berg JP, Nakstad B. The role of plasma and urine metabolomics in identifying new biomarkers in severe newborn asphyxia: a study of asphyxiated newborn pigs following cardiopulmonary resuscitation. PLoS ONE. (2016) 11:e0161123. doi: 10.1371/journal.pone.0161123

28. Beckstrom AC, Humston EM, Snyder LR, Synovec RE, Juul SE. Application of comprehensive two-dimensional gas chromatography with time-of-flight mass spectrometry method to identify potential biomarkers of perinatal asphyxia in a non-human primate model. J Chromatogr A. (2011) 1218:1899906. doi: 10.1016/j.chroma.2011.01.086

29. Reinke SN, Walsh BH, Boylan GB, Sykes BD, Kenny LC, Murray DM, et al. $1 \mathrm{H}$ NMR derived metabolomic profile of neonatal asphyxia in umbilical cord serum: implications for hypoxic ischemic encephalopathy. J Proteome Res. (2013) 12:4230-9. doi: 10.1021/pr400617m

30. Haiju Z, Suyuan H, Xiufang F, Lu Y, Sun R. The combined detection of umbilical cord nucleated red blood cells and lactate: early prediction of neonatal hypoxic ischemic encephalopathy. J Perinat Med. (2008) 36:2407. doi: 10.1515/JPM.2008.035

31. O'Boyle DS, Dunn WB, O'Neill D, Kirwan JA, Broadhurst DI, Hallberg B, et al. Improvement in the prediction of neonatal hypoxic-ischemic encephalopathy with the integration of umbilical cord metabolites and current clinical makers. J Pediatr. (2021) 229:175-81 e1. doi: 10.1016/j.jpeds.2020.09.065

32. Gjerris AC, Staer-Jensen J, Jorgensen JS, Bergholt T, Nickelsen C. Umbilical cord blood lactate: a valuable tool in the assessment of fetal metabolic acidosis. Eur J Obstet Gynecol Reprod Biol. (2008) 139:1620. doi: 10.1016/j.ejogrb.2007.10.004

33. Solberg R, Enot D, Deigner HP, Koal T, Scholl-Burgi S, Saugstad OD, et al. Metabolomic analyses of plasma reveals new insights into asphyxia and resuscitation in pigs. PLoS ONE. (2010) 5:e9606. doi: 10.1371/journal.pone.0009606

34. da Silva Hennebert SN, Denis R, Wayenberg JL. Clinical value of a single postnatal lactate measurement after intrapartum asphyxia. Acta Paediatr. (2000) 89:320-3. doi: 10.1111/j.1651-2227.2000.tb01334.x

35. Chou YH, Tsou Yau KI, Wang PJ. Clinical application of the measurement of cord plasma lactate and pyruvate in the assessment of high-risk neonates. Acta Paediatr. (1998) 87:764-8. doi: 10.1111/j.1651-2227.1998.tb01744.x

36. Murray DM, Boylan GB, Fitzgerald AP, Ryan CA, Murphy BP, Connolly S. Persistent lactic acidosis in neonatal hypoxic-ischaemic encephalopathy correlates with EEG grade and electrographic seizure burden. Arch Dis Child Fetal Neonatal Ed. (2008) 93:F183-6. doi: 10.1136/adc.2006.10 0800

37. Wu TW, Tamrazi B, Hsu KH, Ho E, Reitman AJ, Borzage M, et al. Cerebral lactate concentration in neonatal hypoxic-ischemic encephalopathy: in relation to time, characteristic of injury, and serum lactate concentration. Front Neurol. (2018) 9:293. doi: 10.3389/fneur.2018.00293

38. Shah S, Tracy M, Smyth J. Postnatal lactate as an early predictor of short-term outcome after intrapartum asphyxia. J Perinatol. (2004) 24:1620. doi: $10.1038 /$ sj.jp.7211023

39. Rey-Santano C, Mielgo VE, Gastiasoro E, Murgia X, Lafuente H, RuizDel-Yerro E, et al. Early cerebral hemodynamic, metabolic, and histological changes in hypoxic-ischemic fetal lambs during postnatal life. Front Neurosci. (2011) 5:111. doi: 10.3389/fnins.2011.00111

40. Ikeda T, Murata Y, Quilligan EJ, Parer JT, Theunissen IM, Cifuentes P, et al. Fetal heart rate patterns in postasphyxiated fetal lambs with brain damage. Am J Obstet Gynecol. (1998) 179:1329-37. doi: 10.1016/S0002-9378(98)70156-5
41. Kreisberg RA. Lactate homeostasis and lactic acidosis. Ann Intern Med. (1980) 92:227-37. doi: 10.7326/0003-4819-92-2-227

42. Stacpoole PW, Wright EC, Baumgartner TG, Bersin RM, Buchalter S, Curry SH, et al. Natural history and course of acquired lactic acidosis in adults. DCA-Lactic Acidosis Study Group. Am J Med. (1994) 97:4754. doi: 10.1016/0002-9343(94)90047-7

43. Ando M, Shimizu K. [Acute renal failure with lactic acidosis]. Nihon Jinzo Gakkai Shi. (1990) 32:729-37.

44. Stanley WC, Mazer CD, Neese RA, Wisneski JA, Cason BA, Demas KA, et al. Increased lactate appearance and reduced clearance during hypoxia in dogs. Horm Metab Res. (1990) 22:478-84. doi: 10.1055/s-2007-1004951

45. Frey B, Pfenninger J, Bachmann D. Prognostic value of hyperlactatemia in neonates. J Pediatr. (1995) 127:334. doi: 10.1016/S0022-3476(95)70332-2

46. Pineiro-Ramos JD, Nunez-Ramiro A, Llorens-Salvador R, Parra-Llorca A, Sanchez-Illana A, Quintas G, et al. The Hypotop Study, metabolic phenotypes of hypoxic-ischemic encephalopathy with normal vs. pathologic magnetic resonance imaging outcomes. Metabolites. (2020) 10:109. doi: 10.3390/metabo10030109

47. Ahearne CE, Denihan NM, Walsh BH, Reinke SN, Kenny LC, Boylan GB, et al. Early cord metabolite index and outcome in perinatal asphyxia and hypoxic-ischaemic encephalopathy. Neonatology. (2016) 110:29602. doi: 10.1159/000446556

48. Longini M, Giglio S, Perrone S, Vivi A, Tassini M, Fanos V, et al. Proton nuclear magnetic resonance spectroscopy of urine samples in preterm asphyctic newborn: a metabolomic approach. Clin Chim Acta. (2015) 444:250-6. doi: 10.1016/j.cca.2015.02.004

49. Locci E, Noto A, Puddu M, Pomero G, Demontis R, Dalmazzo C, et al. A longitudinal. 1H-NMR metabolomics analysis of urine from newborns with hypoxic-ischemic encephalopathy undergoing hypothermia therapy. Clinical and medical legal insights. PLoS ONE. (2018) 13:e0194267. doi: 10.1371/journal.pone.0194267

50. Noto A, Pomero G, Mussap M, Barberini L, Fattuoni C, Palmas F, et al. Urinary gas chromatography mass spectrometry metabolomics in asphyxiated newborns undergoing hypothermia: from the birth to the first month of life. Ann Transl Med. (2016) 4:417. doi: 10.21037/atm.2016.11.27

51. Hanrahan JD, Cox IJ, Edwards AD, Cowan FM, Sargentoni J, Bell JD, et al. Persistent increases in cerebral lactate concentration after birth asphyxia. Pediatr Res. (1998) 44:304-11. doi: 10.1203/00006450-199809000-00007

52. van Cappellen AM, van Walsum Jongsma HW, Wevers RA, Nijhuis JG, Crevels J, Engelke UF, et al. Hypoxia in fetal lambs: a study with (1)HMNR spectroscopy of cerebrospinal fluid. Pediatr Res. (2001) 49:698704. doi: 10.1203/00006450-200105000-00015

53. Sarafidis K, Efstathiou N, Begou O, Soubasi V, Agakidou E, Gika E, et al. Urine metabolomic profile in neonates with hypoxic-ischemic encephalopa-thy. Hippokratia. (2017) 21:80-4.

54. Denihan NM, Kirwan JA, Walsh BH, Dunn WB, Broadhurst DI, Boylan GB, et al. Untargeted metabolomic analysis and pathway discovery in perinatal asphyxia and hypoxic-ischaemic encephalopathy. J Cereb Blood Flow Metab. (2019) 39:147-62. doi: 10.1177/0271678X17726502

55. Walsh BH, Broadhurst DI, Mandal R, Wishart DS, Boylan GB, Kenny LC, et al. The metabolomic profile of umbilical cord blood in neonatal hypoxic ischaemic encephalopathy. PLoS ONE. (2012) 7:e50520. doi: 10.1371/journal.pone.0050520

56. El-Farghali OG, El-Chimi MS, El-Abd HS, El-Desouky E. Amino acid and acylcarnitine profiles in perinatal asphyxia: a case-control study. J Matern Fetal Neonatal Med. (2018) 31:1462-9. doi: 10.1080/14767058.2017.1319354

57. Solberg R, Kuligowski J, Pankratov L, Escobar J, Quintas G, Lliso I, et al. Changes of the plasma metabolome of newly born piglets subjected to postnatal hypoxia and resuscitation with air. Pediatr Res. (2016) 80:28492. doi: $10.1038 /$ pr.2016.66

58. McAdams RM, McPherson RJ, Kapur RP, Juul SE. Focal brain injury associated with a model of severe hypoxic-ischemic encephalopathy in nonhuman primates. Dev Neurosci. (2017) 39:107-23. doi: 10.1159/000456658

59. Fatemi A, Wilson MA, Johnston MV. Hypoxic-ischemic encephalopathy in the term infant. Clin Perinatol. (2009) 36:835-58. doi: 10.1016/j.clp.2009.07.011

60. Ferriero DM. Neonatal brain injury. N Engl J Med. (2004) 351:198595. doi: 10.1056/NEJMra041996 
61. Lou BS, Wu PS, Liu Y, Wang JS. Effects of acute systematic hypoxia on human urinary metabolites using LC-MS-based metabolomics. High Alt Med Biol. (2014) 15:192-202. doi: 10.1089/ham.2013.1130

62. DuPont TL, Chalak LF, Morriss MC, Burchfield PJ, Christie L, Sanchez PJ. Short-term outcomes of newborns with perinatal acidemia who are not eligible for systemic hypothermia therapy. J Pediatr. (2013) 162:3541. doi: 10.1016/j.jpeds.2012.06.042

63. Prempunpong C, Chalak LF, Garfinkle J, Shah B, Kalra V, Rollins N, et al. Prospective research on infants with mild encephalopathy: the PRIME study. J Perinatol. (2018) 38:80-5. doi: 10.1038/jp.2017.164

64. Walsh BH, Neil J, Morey J, Yang E, Silvera MV, Inder TE, et al. The frequency and severity of magnetic resonance imaging abnormalities in infants with mild neonatal encephalopathy. J Pediatr. (2017) 187:26-33 e1. doi: 10.1016/j.jpeds.2017.03.065

65. Sanchez-Illana A, Thayyil S, Montaldo P, Jenkins D, Quintas G, Oger C, et al. Novel free-radical mediated lipid peroxidation biomarkers in newborn plasma. Anal Chim Acta. (2017) 996:88-97. doi: 10.1016/j.aca.2017.09.026

66. Farooqui AA, Horrocks LA, Farooqui T. Glycerophospholipids in brain: their metabolism, incorporation into membranes, functions, and involvement in neurological disorders. Chem Phys Lipids. (2000) 106:1-29. doi: 10.1016/S0009-3084(00)0 0128-6
67. Liu J, Sheldon RA, Segal MR, Kelly MJ, Pelton JG, Ferriero DM, et al. $1 \mathrm{H}$ nuclear magnetic resonance brain metabolomics in neonatal mice after hypoxia-ischemia distinguished normothermic recovery from mild hypothermia recoveries. Pediatr Res. (2013) 74:170-9. doi: 10.1038/pr.2013.88

68. Prins ML. Cerebral metabolic adaptation and ketone metabolism after brain injury. J Cereb Blood Flow Metab. (2008) 28:L116. doi: $10.1038 /$ sj.jcbfm. 9600543

69. Chu CY, Xiao X, Zhou XG, Lau TK, Rogers MS, Fok TF, et al. Metabolomic and bioinformatic analyses in asphyxiated neonates. Clin Biochem. (2006) 39:203-9. doi: 10.1016/j.clinbiochem.2006.01.006

Conflict of Interest: The authors declare that the research was conducted in the absence of any commercial or financial relationships that could be construed as a potential conflict of interest.

Copyright (c) 2021 Debuf, Carkeek and Piersigilli. This is an open-access article distributed under the terms of the Creative Commons Attribution License (CC BY). The use, distribution or reproduction in other forums is permitted, provided the original author(s) and the copyright owner(s) are credited and that the original publication in this journal is cited, in accordance with accepted academic practice. No use, distribution or reproduction is permitted which does not comply with these terms. 